\title{
Impact of Covid-19 Outbreak Pandemic on the Radiotherapy Practice: A Survey Study of Indian States
}

\author{
Vindhyavasini Prasad Pandey ${ }^{1}$, Teerthraj Verma ${ }^{2}$
}

${ }^{1}$ Department of Medical Physics, Hind Institute of Medical Sciences, Safedabad, Barabanki, UP- 225003, India. ${ }^{2}$ Department of Radiotherapy, King George's Medical University, UP Lucknow, India.

\begin{abstract}
Background \& objectives: Immunocompromised cancer patients are venerable for the infection and so the professionals involved in radiotherapy too. The objective of this online survey was to assess the impact of Covid-19 on radiotherapy practices, safety measures taken by the radiotherapy professionals during the peak of Covid-19 infection in India. Methods: Structured questionnaire was shared online to assess the awareness and knowledge pertaining to safety of protective items, importance and method of screening, QA of the instruments, safety measures taken in radiotherapy department, methods of the sanitization, use of mask and methods of Covid-19 waste managements. Results: Survey results indicated that almost all participants (97.4\%) were aware of the severity of Covid-19 virus. In safety at the individual level, $51.3 \%$ of the staff reported changing hand gloves before handling new patients and $41 \%$ did not follow the same. In this survey, regarding working days (in a week), employees were working for six days in $56.4 \%$ centres, $28.2 \%$ centres for three days of a week and remaining $15.4 \%$ centres were operational with five days of week. At $38.5 \%$ centres, radiotherapy personals found infected with the virus however large number of centres $(53.8 \%)$ revealed that so far, no radiotherapy professionals got infected. Interpretation \& conclusions: The current survey comprised 31 questions pertaining to various aspects of radiotherapy practices and radiotherapy professional safety from Covid infections and changes followed due to Covid-19 epidemic. The individual professional's inputs could help to deal with such epidemic in even better way.
\end{abstract}

Keywords: Covid-19- Medical Physicist- QA amid Covid-19- Radiotherapy Practices- Sanitization

Asian Pac J Cancer Care, 6 (Suppl 1), 35-40

Submission Date: 06/26/2021 Acceptance Date: 08/25/2021

\section{Introduction}

World health Organization (WHO) decided to declare Covid-19, never seen before contagious diseases, as epidemic at the global level, on 30 Jan 2020 after almost a month later from the date of first case of such unexplained pneumonia was observed. Initially, on the $30^{\text {th }}$ Dec, red-headed document was issued by the Wuhan Municipal health committee on the treatment of this unknown pneumonia [1]. Epidemic intelligence of WHO trapped the news of patients of "Viral Pneumonia" in People's republic of china from the open sources on Dec $31^{\text {st }} 2019$. This deadly virus disseminated itself in very fast way affecting every sector of society be it industry, education, health, employments, farming and many more. The destruction amplitude got heights many folds in terms of increased cases and fatalities on each passing day since broke out of this infection.

It was the "social distancing" measure which was the first officially suggested method to restrict the mobility of Covid -19. Unlike other countries, besides big leap in number of cases, India took a big step to roll out the nationwide complete lockdown when only 550 cases were reported. This was assumed to be a final jump to come out with "virus contained" India. However, as never before seen experience, it took to extend the lockdown which contributed immensely in deterioration of all the sectors of life and growth developments including the health care infrastructure. The speed of spread of this ever-new respiratory infection Covid-19 could be imagined by

Corresponding Author:

Dr. Teerthraj Verma

Department of Radiotherapy, King George's Medical University, UP Lucknow 226003, India.

Email: teerth05kashi@gmail.com 
the WHO action time line that within almost a month of time, this apex global body had to issue a highest level of alarm in concern of international health emergency [2]. ON March 08, 2020, more than the 100,000 were found infected belonging to 100 countries. It was less than two and half months on March 08, 2020, WHO declared the Covid-19 as "Global Pandemic".

These actions of the apex bodies without a precise explanation of the cause and treatment, fueled the fear of contagion and the fire of myths. Professionals involved in the management of diseases were also not significantly out of its reach as increasing number of cases in this community and deaths were reported from other parts of the world including India $[3,4]$.

Very soon it was experienced that that cancer patients are not only very infection prone but also have least favorable responses. Being of infectious nature, Covid-19 affected many cancer centers, including limited or delayed treatment facilities. However, the interventions and guidelines provided for such environments helped to invent the new normal and new aspects adding to the routine. It is well known that cancer patients are of low immunity and in almost all cancers delay in treatment could greatly worsen the outcome and quality as well. [5]. In the present era, radiotherapy, is found to be one of the major piler in the management of the cancer diseases and almost $50 \%$ of the cancer patients needs radiotherapy as a part of treatment other than surgery, chemotherapy, immunotherapy etc. As the responsible health care provider, the radiotherapy of cancer patients could not be interrupted or discontinued in presence of these odd full circumstances however a wave of dilemma was observed in hidden way. Therefore, need was observed to record the exact status of different aspects among cancer health professionals involved in cancer patient's radiotherapy managements.

The purpose of this online survey was to demonstrate the impact of Covid-19 on radiotherapy practices and radiotherapy professionals during the peak of Kovid-19 infection in India.

\section{Materials and Methods}

An online questionnaire was formatted in Google forms available freely. This was a structured survey for the qualitative assessment of different aspects impacted by the Covid-19 virus. The questionnaire was designed to get the estimate of level of changes followed in routine radiotherapy workflow in this new environment. And patient managements.

The questionnaire had welcome address and background for the respondents. There were few of the options for individual details like name, role, affiliation and address to have spatial and geographical details of the respondent. At the end of survey questionnaire, a message was conveyed the details provided would only be used in scientific publication only and would not be shared else.

The link of the questionnaire was circulated among the radiotherapy personals such as radiation oncologists, medical physicists, clinical and physics residents and radiotherapy technologists with a request to provide their opinion/answers. It was shared with almost fifty radiotherapy professional (RTP) and 38 responses were received in the data base directly. The survey was conducted during June, 2020 when Covid-19 cases were continuously in upward direction of its growth. In the period of survey 8.6.2020 to 23.10.2020, each passing day had witnessed record number of infected cases as well as in causalities.

Total 31 questions were shared online to assess the entire length and breaths of the radiotherapy practice as for as radiation delivery without compromising the accuracy - precision with utmost safety of the patients, attendant and personal. The respondents of the survey includes both from the government and private radiotherapy centers. The questions were to assess the prevailing awareness and knowledge pertaining to the safety such as availability of protective items, use of mask sanitizers and PPE kit; importance and method of screening like sensitivity of prevailing testing methods; safety measures taken in radiotherapy department and out patients departments, methods of the sanitization such as fumigation frequency, mopping and sanitization of the ray cost and couch, use of mask Covid help desk and methods of Covid waste management, which had been one of the major challenges in the Covid-19 treatment and managements.

\section{Results}

A total of thirty-one questions from various aspects, as shown in Figure 1, were included in this online survey. Thirty-nine radiotherapy personal belonging to 13 states/ UTs, viz Chhattisgarh, Uttarakhand, Chandigarh, Haryana, Uttar Pradesh, Maharashtra, Madhya Pradesh, Delhi, Punjab, Kerala, Bihar, Andhra Pradesh, West Bengal, Tamil Nadu, participated in this open survey. These participating departments have a wide spectrum of cancer treatment infrastructures and modalities ranging from conventional to state -of-the-art technology in the field of cancer treatment. In addition, some of the radiotherapy centers are dealing with huge patient load e.g., TMH, Mumbai and AIIMS, New Delhi.

The study planning of this survey was to collect the

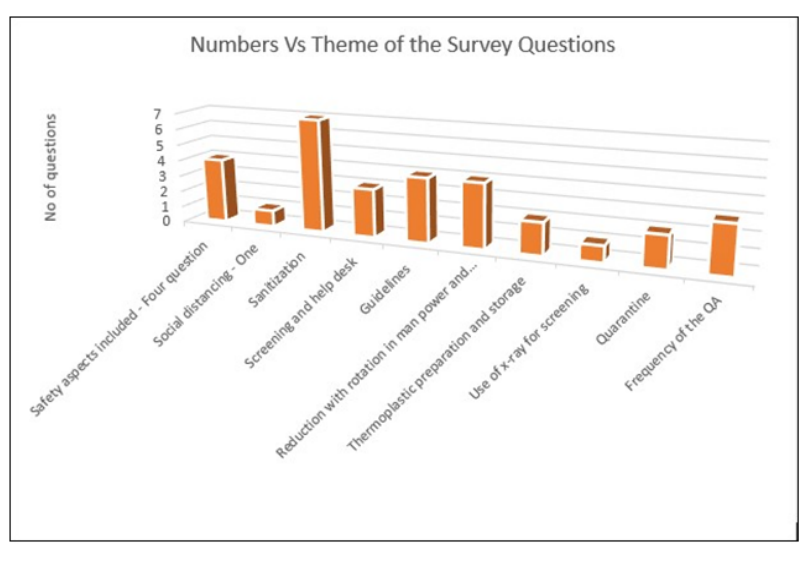

Figure 1. Number of Questions from Various Aspects Covered in the Survey Questionnaire 
Table 1. Survey Question and Responses by the Participants

\begin{tabular}{llccc}
\hline Sr. No. & Survey Question & \multicolumn{3}{c}{ Response (\%) } \\
& & Yes & No & May be \\
\hline 1 & Awareness of Covid severity among participants & 97.4 & 2.6 & 0 \\
2 & Awareness of MoFHW guidelines & 100 & 0 & 0 \\
3 & Thermal scanning used by professionals & 71.8 & 17.9 & 10.3 \\
4 & Ratio of hospitals providing PPE kit & 61.4 & 25.6 & 10.3 \\
5 & Ratio of patients using mask while visiting to department & 100 & 0 & 0 \\
6 & Availability of sanitiser at premises & 69.2 & 23.1 & 7.7 \\
7 & Ratio of professionals using gloves & 41 & 51.3 & 7.7 \\
8 & Senitisation of treatment couch adopted by different participants & 63.2 & 21.1 & 15.8 \\
9 & Change in work practise & 71.1 & 18.4 & 10.5 \\
10 & Senitisation of patient mask & 30.8 & 51.3 & 17.9 \\
11 & \% of centres that made CT chest of the patient mandatory to visit the department & 68.4 & 23.7 & \\
12 & Response of the RP regarding change of CT syringe & 53 & 40.8 & 6.12 \\
13 & Ratio of centres followed work force reduction & 76.9 & 17.9 \\
14 & QA performed during Covid & 68.4 & 18.4 & 13.2 \\
15 & Use of Gloves and sanitizer during QA & 64.9 & 21.6 & 13.5 \\
16 & RP contracted at radiotherapy facility & 38.5 & 53.8 & 2.6
\end{tabular}

responses from each types of professionals viz radiation oncologists (RO), medical physicists (MP), radiotherapy technologists (RTT), dosimetrists and PG students (Clinical and Physics) involved in the management of radiation delivery. From the survey results, it was found that the MP found with highest $(51.3 \%)$ response as compared to RO $(25.6 \%)$ and RTT $(17.9 \%)$, with the lowest contribution by dosimetric and PG students of almost equal amount, as shown in Figure 2.

Theses professional, maximally, belonged to the government radiation oncology departments (69.2\%), $28.2 \%$ private hospitals and remaining from the research centers and semi government centers (Figure 3).

This study was initiated in mid of the June, almost after 5 months later from the first confirmed case reported in Kerala (India) on Jan 27, 2020. And in this period of time, awareness of Covid was a major approach for virus prevention without compromising the safety of professionals. Survey results indicate that almost all participants $(97.4 \%)$ were aware of the severity of this notorious virus except for a few participants (Table 1, Sr No.1).

Probably, it was due to the availability of guidelines provided by the Ministry of Health and Family Welfare for the precautions to be taken during COVID-19 pandemic by $100 \%$ of the participants. Nevertheless, despite this awareness among RPs, $25.6 \%$ of the personalities were found to be fearful and $53.8 \%$ expressed no fear, but $25.6 \%$ were not sure of this (Table 1, Sr No. 2). As a safeguard to prevent the spread of the virus, "social distancing and infra-red thermal scanning" have been promoted among the general public since the early days of reporting as viruses and such of harmful characters destructive on an unimaginable level. Similarly, as shown in Table 1 sr. no 3, participating radiotherapy centres communicated that 71.8 $\%$ were following this practice and $17.9 \%$ were not doing thermal screening of the patients while allowing them in the radiation delivery room while $10.3 \%$ personal were unaware about this safety method.

Also, $64.1 \%$ were provided with the appropriate

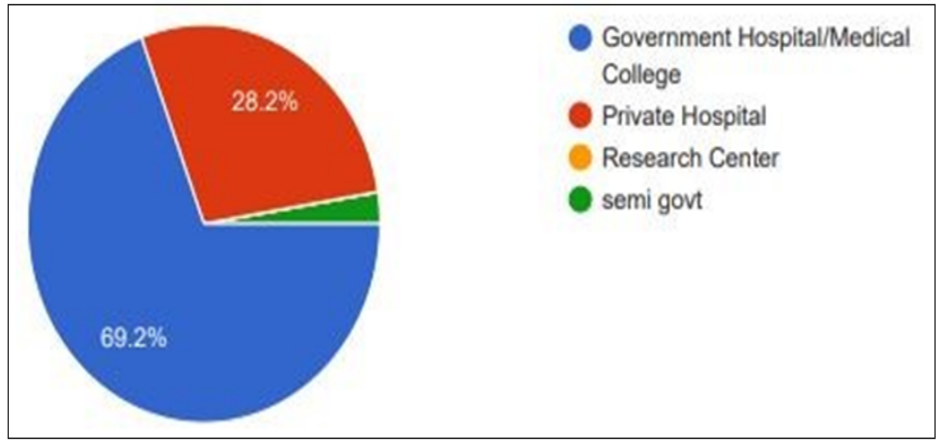

Figure 2. Participants Institute 


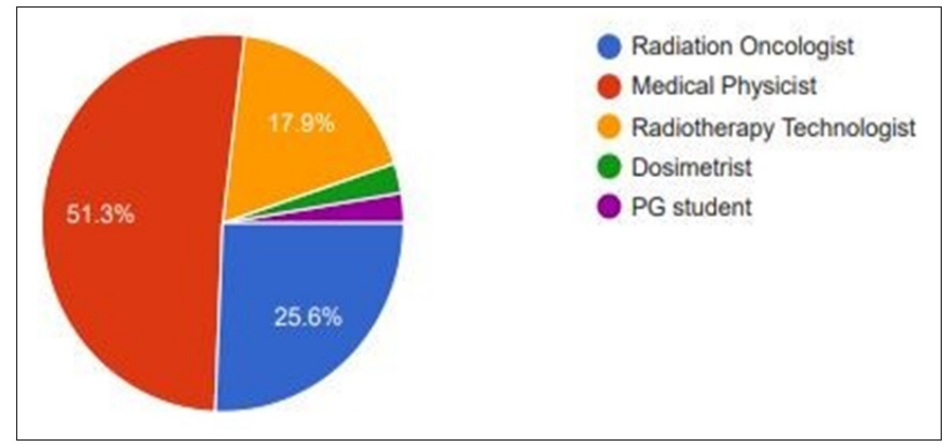

Figure 3. Type of Professionals Participated in Survey

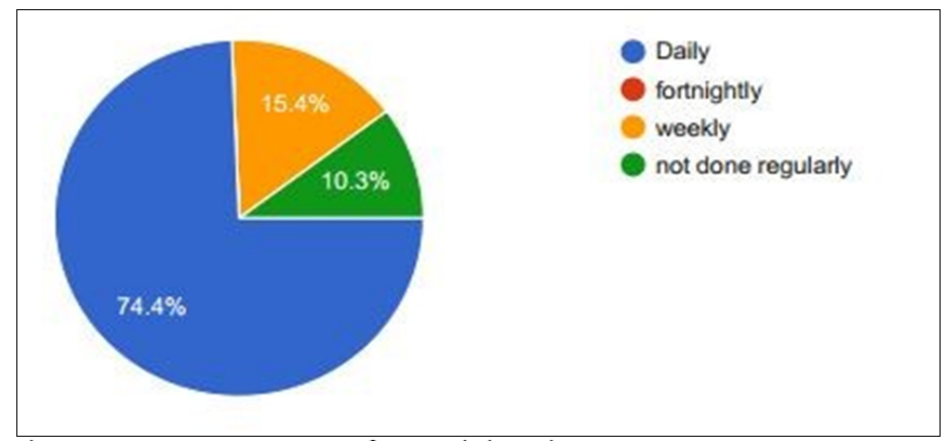

Figure 4. Frequency of Sensitization

safety kit by the hospital and not $25.6 \%$ while 10.3 did not insist on bringing it to clinical work regularly (Table 1, Sr. No. 4). Together with much safety ads by the personal, $100 \%$ patients and their attendants were found wearing mask while visiting the hospital including the treatment room as presented in Table 1, Sr. No. 5.

Together with this, $69.2 \%$ participants conveyed that sanitiser was made available to the patients and their attendants at the entry and exit of the department premise (s) as mentioned in Table 1, Sr. No.6.

For the safety at individual level, $51.3 \%$ of staff reported changing hand gloves before handling new patients and $41 \%$ did not follow the same (Table 1, Sr. No. 7).

The protocol followed for the safety of work place were also assessed. In this context, $63.2 \%$ participants inform about the sanitisation of treatment couch after every patient, in contrast to $21 \%$ where this was not adopted and around $16 \%$ were randomly doing this as demonstrated in Table 1, Sr No. 8.

About $75 \%$ of the treatment facilities were reported to be sanitised daily whereas weekly, $15.4 \%$ facilities and about $10.3 \%$ followed it very randomly (Figure 4 ).

Besides this, they share that most of the facility (59\%) uses mopping with the disinfectant for the sanitisation though fumigation (23\%) and sanitizer spray (17.9\%) is also done for this purpose (Figure 5). For the matter of change in work practice in the routine workflow in view of prevailing pandemic, $71 \%$ personal informed about maintaining the distance among the thermoplastic masks of the patients, opposite to $18 \%$ who were not doing this way (Table 1, Sr. No. 9).

Not only that, $52 \%$ shared that they demonstrated sensitization of the thermoplast before using it on patients, while about $37 \%$ did not do so and about $10 \%$ were not sure of the same (Table 1, Sr. No. 10).

At the patient level, as the additional safety, around

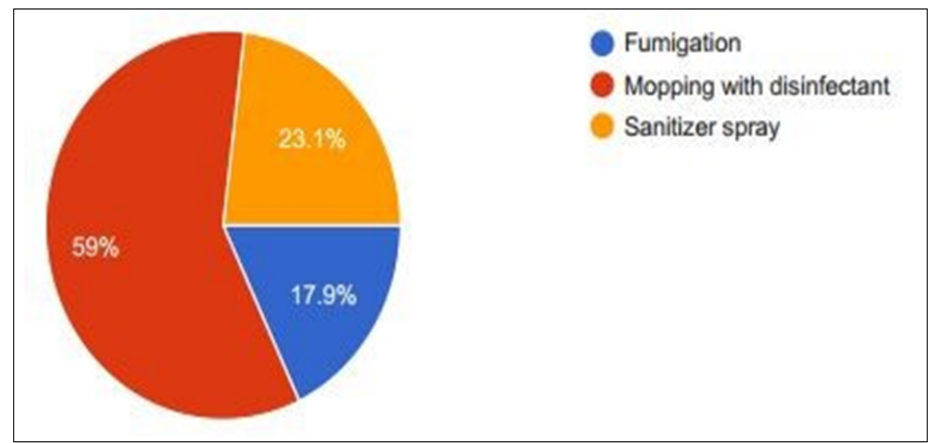

Figure 5. Mode of Senitisation of Sites 


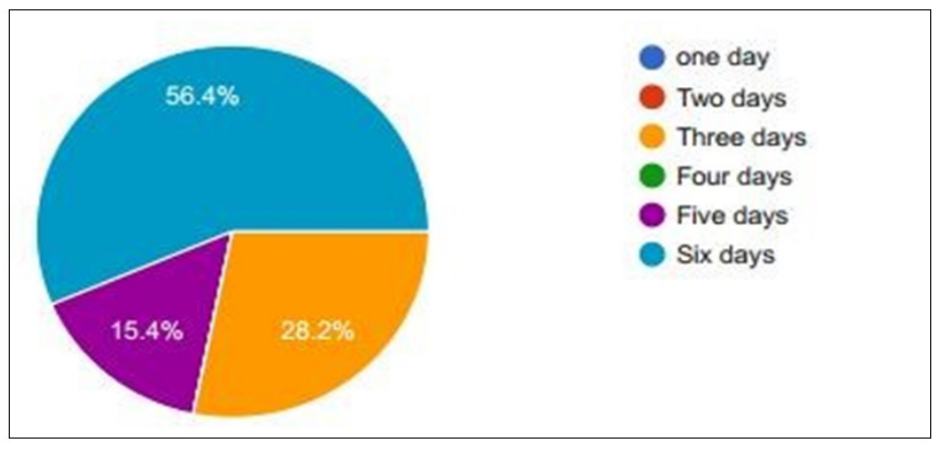

Figure 6. Working Days in a Week

one third of the participating centres $(30.8 \%)$ made mandatory to produce patient chest $\mathrm{x}$-ray before starting of the treatments however, half of the centres stand opposing these requirements and $17.9 \%$ centres followed safety tool randomly according to input as shown in Table 1, Sr. No. 11.

This may be the result of innovative approaches, self-learning, and guidelines provided by stakeholders, which have changed as routine workflows to prevent transition concerns. Even during CT simulation, which is now becoming very common, $68.4 \%$ centres started using fresh tubing/syringe for each of the new patient during the simulation though $23.7 \%$ centres did not follow so (Table 1, Sr. No. 12).

Also, the practice of work force reduction, at work stations, was followed promoting the work from home remotely to plan a treatment planning only by few of the centres $(18 \%)$ in contrast to $76.9 \%$ which did not follow the such strategy for the reduction in man powers (Table 1, Sr. No. 13). Also, in the survey information regarding working days (in a week) of the individual employees were requested which shows that individual employees were working for six days of week in $56.4 \%$ centres, for three days of week in $28.2 \%$ and remaining $15.4 \%$ centres were operation with Five day of week working pattern (Figure 6).

In an environment fraught with virus, where contact with living and non-living and vice-versa is not at all safe, it can be understood how difficult the QA function is, which becomes manifold infection-prone due to its frequent operations. Nevertheless, surprisingly, $68.4 \%$ of centres performed daily, weekly, and monthly QA performed during the Covid-19 epidemic, with relatively few (18.4\%) who did not comply as informed in Table 1, Sr. No. 14. Further, details add that $64.9 \%$ centres used the gloves and sanitizer to clean QA equipment and $21.6 \%$ shared that they did not used gloves and sanitiser to clean the QA equipment (Table 1, Sr. No. 15).

And, as the last query in survey questionnaire, it was found that radiotherapy personal at $38.5 \%$ centres have been contracted with the virus however, large number of centres $(53.8 \%)$ revealed that so far, no RP got infected. Nonetheless, participants from $7.7 \%$ centres could not confirmed either case as mentioned in Table 1, Sr. No. 16.

\section{Discussion}

Today, in the era of modern technology, radiotherapy is becoming essential for cancer treatment and management. It could be used for both palliative and therapeutic intentions, both. Thus, this has influenced both government officials, particularly the ministries of health, and upcoming medical professionals in general in opting the field of radiation oncology from a career and employment standpoint [5]. In this present study, radiotherapy departments belonging to all regions except North-East region participated. Munshi et al in their study revealed that North-East regions contributes around $3.57 \%$ population wise and $7.8 \%$ burden of total area with lowest number of telecobalt and LINACs functional in this region. Also, highest contribution $(25 \%)$ in total population of India is from North India region. Therefore, the data of present survey could be taken as pan India radiotherapy representative results [6].

The report from the ICMR and NCDIR gives an estimate of 14.0 lakh cancer patients by 2020 released in 2018 and as mentioned in previous paragraph around 7 lakh patients $(50 \%)$ would be visiting the radiotherapy facility all over the country. In terms of numbers of personals involved in each of the radiotherapy facilities, a large part of the health professionals is associated with this specialty and community. Therefore, it was logical to conduct such survey which would surely help in fight of such future disasters as well as analyzing the methodology currently adopted in such epidemics to strengthen the health facilities at present $[7,8]$.

The timing of the survey is also important. The questionnaire was carried out on-air when the Covid-19 cases were continuously in the direction of its development. During the period of the survey i.e., from 8.6.2020 to 23.10.2020, not only the number of infected cases was recorded in each passing day, but also the record of deaths. The number of corona cases was steadily increasing from January to September, earning the maximum record in its credit, and it began to go down since October. In these times, things were not so clear and each day we had to add new guidelines, abandoning the concept of social design, including the use of sanitizer, its use in mopping for surface sanitization and fumigation for disinfection and prevention. As clear from the survey results, which is derived, from the data collected during 
the initial months (before reaching to peak) as practiced that time itself, also that maximum of the participants followed social distancing, sanitization, use of mask and implemented the same for visitors including the patients. It is now an established method of stopping the spread of the virus after one year of past. The present survey was conducted during Jun - Oct 2020 and during this time new safety, precaution guidelines from MoHFW and ICMR were introduced daily not only for the prescription but also for the laboratory personal, also [9]. Apart from these, one of the important tasks was to manage the wastes coming out during in ward treatment, procedures and also from the quarantine patients. It was the awareness of the new guidelines for BMW amid Covid -19 which played a crucial role in conforming the virus. Probably it has been one of the reason India had not seen that much infections and causalities in comparison to other parts of the globe and then expected and projected as it was at the time of starting of the survey. Over the period of Jan 2020 to Sep 2020 it was continuously on surge every month. It is also evident and supported by the survey response on the use of proper bin for the handling of the waste generated by the department of radiotherapy. About $97.4 \%$ of the facilities responded indicating that their department is equipped with appropriate bin arrangements. It is a matter of cancer due to reason of contagious nature of virus and increased load as a result of use of PPE kit, mask, gloves sanitizer etc., which, if not handled properly could be path for propagation of virus. It is matter of appreciation that other than existing guidelines, radiotherapy facilities adopted the newly provided rules for the management of wastes such as gloves, mask, PPE kit and similar protective equipments which are used in Covid holding areas, wards, procedure rooms etc. According to new guidelines, management of these wastes requires separate place together with the use of dedicated bins only for these wastes (Table 1, Sr. No. 17).

A double layer poly bag policy was advised to place in the bin/container preferably zipped one and each time when poly bag (containing waste) is removed from the bin/ container, it has to be sanitized using the proper chemical solution spray. Most of the infectious waste required to be put in the yellow bin/container. It was need of the hour, as already overburdened with the Non-covid bio medical waste (BMW). India generated 3000 tones waste in June and this increased in months of July to 4000 tones maintained the surge continuously till September with a spike in BMW load of 5400 tone [10].

Apart from this, no doubt initially most of the professional found to be freighted of this deadly virus including general public though patient load was not that much reduced as $59 \%$ professional communicated about the reduction in patient load as shown in Table 1, Sr. No. 18.

\section{Acknowledgements}

The authors thank the participants for their valuable responses in online survey questionnaire.

Financial support \& Sponsorship

None

Conflicts of interest

NIL

\section{References}

1. https://promedmail.org/promed-post $/ \mathrm{id}=6864153 \% 20$ \#COVID19 (accessed on February 3, 2021).

2. https://www.who.int/emergencies/diseases/novelcoronavirus-2019/interactive-timeline\#! (accessed on February 3, 2021).

3. https://pib.gov.in/PressReleseDetailm.aspx?PRID=1614215 (accessed on February 3, 2021).

4. https://thewire.in/Covid-19-india-timeline (accessed on February 3, 2021).

5. Pramesh C, Badwe RA. Cancer Management in India during Covid-19. New England Journal of Medicine. 202005 14;382(20):e61. https://doi.org/10.1056/nejmc2011595

6. Munshi A, Ganesh T, Mohanti B. Radiotherapy in India: History, current scenario and proposed solutions. Indian Journal of Cancer. 2019;56(4):359. https://doi.org/10.4103/ ijc.ijc $82 \quad 19$

7. Reuter-Oppermann M, Müller-Polyzou R, Wirtz H, Georgiadis A. Influence of the pandemic dissemination of COVID-19 on radiotherapy practice: A flash survey in Germany, Austria and Switzerland. Chun S. PLOS ONE. 2020 05 21;15(5):e0233330. https://doi.org/10.1371/journal. pone. 0233330

8. https://www.thegreenjournal.com/article/S01678140(20)30303-0/fulltext (accessed on February 3, 2021).

9. https://www.ncdirindia.org/All_Reports/Report_2020/PB/ Press_release.pdf. (accessed date - 03/02/2021).

10. https://cpcb.nic.in/ (accessed on February 3, 2021).

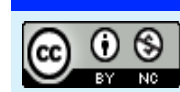

This work is licensed under a Creative Commons AttributionNon Commercial 4.0 International License. 